\title{
AN INEQUALITY CONCERNING THE SMALLEST DISC THAT CONTAINS THE SPECTRUM OF AN OPERATOR
}

\author{
GERHARD GARSKE
}

\begin{abstract}
Any bounded linear operator $T$ on a Hilbert space satisfies the inequality $\sup _{\|x\|=1}\left\{\|T x\|^{2}-|(T x, x)|^{2}\right\} \geqslant R_{T}^{2}$, where $R_{T}$ is the radius of the smallest disc containing the spectrum of $T$. An example is given, which shows that we cannot expect equality for $G_{1}$ operators in general, contrary to a conjecture of Istrătescu.
\end{abstract}

1. For a bounded linear operator $T$ on a Hilbert space $\mathcal{H}$ let $\sigma(T)$ denote its spectrum, $r(T)$ its spectral radius, i.e. $r(T):=\sup \{|\lambda| \mid \lambda \in \sigma(T)\}, W(T)$ its numerical range, and $w(T)$ its numerical radius, i.e. $w(T):=\sup \{|\lambda| \mid \lambda \in W(T)\}$. $T$ is called normaloid, iff $\|T\|=r(T)=w(T)$, and transloid, iff $T-\lambda I$ is normaloid for each $\lambda \in \mathbf{C}$. $T$ is called convexoid, iff $\overline{W(T)}=\operatorname{conv} \sigma(T)$. $(\bar{X}$ denotes the closure of the set $X \subseteq \mathbf{C}$ and conv $X$ its convex hull.) $T$ has the property $G_{1}$, iff $\left\|(T-\lambda I)^{-1}\right\|=1 / d(\lambda, \sigma(T))$ for each $\lambda \in \mathbf{C} \backslash \sigma(T) .(d(\lambda, X)$ denotes the distance between $\lambda$ and the set $X \subseteq \mathbf{C}$.)

Björck and Thomée have shown [1] that normal operators have the property

$$
\sup _{\|x\|=1}\left\{\|T x\|^{2}-|(T x, x)|^{2}\right\}=R_{T}^{2}
$$

where $R_{T}$ is the radius of the smallest disc containing $\sigma(T)$. This equality and especially the left side has a geometrical interpretation: For $\|x\|=1$ we have

$$
\|T x\|^{2}-|(T x, x)|^{2}=\|T x-(T x, x) x\|^{2},
$$

and $T x-(T x, x) x$ is the perpendicular from $T x$ to $x$. Hence for normal operators $R_{T}$ is the supremum over the lengths of all such perpendiculars.

Istrătescu [3] has shown that this is also true for transloid operators. He noticed that, in his proof, the proof of the inequality

$$
\sup _{\|x\|=1}\left\{\|T x\|^{2}-|(T x, x)|^{2}\right\} \geqslant R_{T}^{2}
$$

only depends on properties, which, according to Hildebrandt [2], convexoid operators posses, too. So convexoid operators fulfill at least (2).

In this note we shall prove that (2) is valid for any bounded linear operator.

Istrăţescu additionally conjectured that (1) is also valid for convexoid operators and for operators with the property $G_{1}$. Sheth [4] has shown that this is not true for convexoid operators, and we will give an example of a $G_{1}$ operator, which does not fulfill (1).

Received by the editors July 11, 1978 and, in revised form, April 30, 1979.

AMS (MOS) subject classifications (1970). Primary 47A10; Secondary 47B20.

Key words and phrases. Spectrum, $G_{1}$ operator. 
2. THEOREM. Any bounded linear operator $T$ has the property (2).

Proof. If we apply to $T$ a linear polynomial $p: t \mapsto \alpha t+\beta(t, \alpha, \beta \in \mathbf{C})$, then the left side as well as the right side of (2) only change by the factor $|\alpha|$. Therefore we may confine ourselves to the case that the smallest disc containing $\sigma(T)$ is the unit disc. Hence we have to show that there exists a sequence $\left\{w_{n}\right\}$ of unit vectors such that

$$
\varlimsup_{n \rightarrow \infty}\left(\left\|T w_{n}\right\|^{2}-\left|\left(T w_{n}, w_{n}\right)\right|^{2}\right) \geqslant 1
$$

The boundary of the unit disc and $\sigma(T)$ have in common either

(i) exactly two points $\kappa, \lambda$, or

(ii) at least three points $\kappa, \lambda, \mu$.

In each case, because of the minimality of the unit disc, we may choose them in such a way that 0 is in their convex hull.

In case (i) we have $\kappa=-\lambda$.

In case (ii) there exist three complex numbers $k, l, m$ such that

$$
|k|^{2}+|l|^{2}+|m|^{2}=1 \text { and }|k|^{2} \kappa+|l|^{2} \lambda+|m|^{2} \mu=0
$$

Moreover at least two of them, say $k$ and $l$, cannot vanish. The arguments will be chosen later.

Since $\kappa, \lambda$, and $\mu$ lie on the boundary of $\sigma(T)$, they belong to the approximate point spectrum. Hence there exist sequences of unit vectors $\left\{x_{n}\right\},\left\{y_{n}\right\},\left\{z_{n}\right\}$ such that $T x_{n}=\kappa x_{n}+o(1), T y_{n}=\lambda y_{n}+o(1), T z_{n}=\mu z_{n}+o(1)$. Here and in the following we generally use $f_{n}=g_{n}+o(1)$ as an abbreviation for $\left\|f_{n}-g_{n}\right\| \rightarrow 0$. Without loss of generality (i.e. by passing to subsequences if necessary) we additionally may assume that the sequences of the mutual scalar products $\left\{\left(x_{n}, y_{n}\right)\right\},\left\{\left(y_{n}, z_{n}\right)\right\},\left\{\left(z_{n}, x_{n}\right)\right\}$ do converge.

For each $n \in \mathbf{N}$ we apply the Gram-Schmidt orthonormalization process to the vectors $x_{n}, y_{n}$, and $z_{n}$. So let

$$
\begin{array}{ll}
\tilde{u}_{n}:=y_{n}-\left(y_{n}, x_{n}\right) x_{n}, & u_{n}:=\frac{\tilde{u}_{n}}{\left\|\tilde{u}_{n}\right\|}, \\
\tilde{v}_{n}:=z_{n}-\left(z_{n}, x_{n}\right) x_{n}-\left(z_{n}, u_{n}\right) u_{n}, & v_{n}:=\frac{\tilde{v}_{n}}{\left\|\tilde{v}_{n}\right\|},
\end{array}
$$

so that $\left\{x_{n}, u_{n}, v_{n}\right\}$ is an orthonormal system for each $n \in \mathbf{N}$.

It is easy to check that, by virtue of the distinctness of $\kappa, \lambda$, and $\mu$, there exist no sequences $\left\{\alpha_{n}\right\},\left\{\beta_{n}\right\}$ in $\mathbf{C}$ such that either $\left\{y_{n}-\alpha_{n} x_{n}\right\}$ or $\left\{z_{n}-\alpha_{n} x_{n}-\beta_{n} y_{n}\right\}$ converge to 0 . Therefore $\left\{\tilde{u}_{n}\right\}$ and $\left\{\tilde{v}_{n}\right\}$ cannot converge to 0 , but they converge because of the convergence of the scalar products. Hence there exist sequences $\left\{a_{n}\right\},\left\{b_{n}\right\},\left\{c_{n}\right\}$ in $\mathbf{C}$ such that

$$
\begin{aligned}
& T u_{n}=a_{n} x_{n}+\lambda u_{n}+o(1), \\
& T v_{n}=b_{n} x_{n}+c_{n} u_{n}+\mu v_{n}+o(1),
\end{aligned}
$$

and from their special shape we see that they converge, too, say to $a, b, c \in \mathbf{C}$ respectively. Thus we have 


$$
\begin{aligned}
& T u_{n}=a x_{n}+\lambda u_{n}+o(1), \\
& T v_{n}=b x_{n}+c u_{n}+\mu v_{n}+o(1) .
\end{aligned}
$$

Now let $w_{n}:=k x_{n}+l u_{n}+m v_{n}$ and fix an arbitrary argument for $m$. We can take this $w_{n}$ also in the case (i), if we set $|k|^{2}=|l|^{2}=\frac{1}{2}$ and $m=0$. Here $u_{n}$ is to be chosen as described before, and for $v_{n}$ we can take any vector. So we treat both cases together now.

We have

$$
\begin{aligned}
\left\|w_{n}\right\|^{2} & =|k|^{2}+|l|^{2}+|m|^{2}=1, \\
T w_{n} & =(k \kappa+l a+m b) x_{n}+(l \lambda+m c) u_{n}+m \mu v_{n}+o(1) .
\end{aligned}
$$

With the aid of (3) it is easy to check

$$
\begin{aligned}
\left\|T w_{n}\right\|^{2}= & 1+|l a|^{2}+|m b|^{2}+|m c|^{2} \\
& +2 \operatorname{Re}(k \kappa \overline{l a}+k \kappa \overline{m b}+l a \overline{m b}+l \lambda \overline{m c})+o(1), \\
\left(T w_{n}, w_{n}\right)= & l a \bar{k}+m b \bar{k}+m c \bar{l}+o(1), \\
\left\|T w_{n}\right\|^{2}-\left|\left(T w_{n}, w_{n}\right)\right|^{2}= & 1+|l a|^{2}\left(1-|k|^{2}\right)+|m b|^{2}\left(1-|k|^{2}\right)+|m c|^{2}\left(1-|l|^{2}\right) \\
& +2 \operatorname{Re} k A(l)+2 \operatorname{Re} l B+o(1),
\end{aligned}
$$

if we set

$$
\begin{aligned}
A(l) & :=\kappa \overline{l a}+\kappa \overline{m b}-m \bar{l}^{2} \bar{a}-|m|^{2} c \overline{b l}, \\
B & :=a \overline{m b}\left(1-|k|^{2}\right)+\lambda \overline{m c} .
\end{aligned}
$$

Hence

$$
\left\|T w_{n}\right\|^{2}-\left|\left(T w_{n}, w_{n}\right)\right|^{2} \geqslant 1+2 \operatorname{Re} k A(l)+2 \operatorname{Re} l B+o(1) .
$$

We now specify the argumensts of $k$ and $l$. If $|l B| \geqslant|k A(l)|$ for all arguments of $l$, we take $\arg l$ such that $\operatorname{Re}(l B /|l|)=|B|$. If $\max _{l}|k A(l)|>|l B|$, we take $\arg l$ such that $|k A(l)|>|l B|$ and $\arg k$ such that $\operatorname{Re}(k A(l) /|k|)=|A(l)|$. In both cases we have

$$
\operatorname{Re} k A(l)+\operatorname{Re} l B \geqslant 0 .
$$

Hence

$$
\left\|T w_{n}\right\|^{2}-\left|\left(T w_{n}, w_{n}\right)\right|^{2} \geqslant 1+o(1)
$$

or

$$
\varlimsup_{n \rightarrow \infty}\left\|T w_{n}\right\|^{2}-\left|\left(T w_{n}, w_{n}\right)\right|^{2} \geqslant 1
$$

3. ExAMPLE. The property $G_{1}$ does not imply the property (1).

Proof. Let $\mathcal{H}$ be a two dimensional Hilbert space with a fixed basis and let $S$ be the linear operator on $\mathcal{H}$ represented by $\left(\begin{array}{ll}0 & 1 \\ 0 & 0\end{array}\right) . S$ is topologically nilpotent, so that $\sigma(S)=\{0\}$.

Let $U$ be a bounded linear operator on a Hilbert space $\mathscr{K}$ with the unit disc as spectrum, $\sigma(U)=\{z \in \mathbf{C}|| z \mid \leqslant 1\}$. For the orthogonal direct sum $A \oplus B$ of any two bounded linear operators $A, B$ on two Hilbert spaces we have (Hildebrandt [2]) 
$\|A \oplus B\|=\max \{\|A\|,\|B\|\}, \quad(A \oplus B)^{-1}=A^{-1} \oplus B^{-1}, \quad \sigma(A \oplus B)=\sigma(A) \cup$ $\sigma(B)$. So, if we set $T:=S \oplus \tau U$ for some $\tau \in \mathbf{R}$ with $\tau \geqslant 0$, we have $\sigma(T)=\{z \in$ C $|z| \leqslant \tau\}$ and $R_{T}=\tau$. Let $x:=\left(\begin{array}{l}9 \\ 1\end{array}\right) \oplus 0$. Then $\|x\|=1, T x=\left(\begin{array}{l}1 \\ 0\end{array}\right) \oplus 0$, and $\|T x\|^{2}$ $-|(T x, x)|^{2}=1$. Hence $T$ fails to have the property (1) for $\tau<1$.

We now show that there exist $\tau<1$ and $U$ such that $T$ is a $G_{1}$ operator. For this sake let $U$ additionally be a $G_{1}$ operator. (For instance each normal operator has this property.) All we have to show is the existence of a $\tau<1$ such that for all $\lambda \in \mathbf{C}$ with $|\lambda|>\tau$

$$
\left\|(S-\lambda I)^{-1}\right\| \leqslant\left\|(\tau U-\lambda I)^{-1}\right\|
$$

since then

$$
\left\|(T-\lambda I)^{-1}\right\|=\left\|(\tau U-\lambda I)^{-1}\right\|=\frac{1}{|\lambda|-\tau}=\frac{1}{d(\lambda, \sigma(T))},
$$

because $\tau U$ is a $G_{1}$ operator if $U$ does. We compute $\left\|(S-\lambda I)^{-1}\right\|$. For $R(a):=\left(\begin{array}{ll}1 & a \\ 0 & 1\end{array}\right)(a \in \mathrm{C})$ it is easy to check that

$$
\|R(a)\|=\frac{1}{2}\left(|a|+\sqrt{4+|a|^{2}}\right) .
$$

Since

$$
(S-\lambda I)^{-1}=\frac{1}{\lambda^{2}}\left(\begin{array}{cc}
-\lambda & -1 \\
0 & -\lambda
\end{array}\right)=-\frac{1}{\lambda} R\left(\frac{1}{\lambda}\right)
$$

we therefore have

$$
\left\|(S-\lambda I)^{-1}\right\|=\frac{1}{2|\lambda|}\left(\frac{1}{|\lambda|}+\sqrt{4+\frac{1}{|\lambda|^{2}}}\right) .
$$

So in order to prove (4) we have to show

$$
\frac{1}{2|\lambda|}\left(\frac{1}{|\lambda|}+\sqrt{4+\frac{1}{|\lambda|^{2}}}\right) \leqslant \frac{1}{|\lambda|-\tau} .
$$

In case $\tau \geqslant 1 / 8$ this is equivalent to

$$
\tau^{2}+|\lambda| \leqslant 2|\lambda| \tau+\tau
$$

and this is valid for $\frac{1}{2} \leqslant \tau<1$. Hence $T$ is a $G_{1}$ operator for such $\tau$ and $U$.

I would like to thank the referee, who found an error in the original computation of $\|R(a)\|$.

\section{REFERENCES}

1. G. Björck and V. Thomee, A property of bounded normal operators in Hilbert space, Ark. Math. 4 (1963), 551-555.

2. S. Hildebrandt, Über den numerischen Wertebereich eines Operators, Math. Ann. 164 (1966), 230-247.

3. V. Istrătescu, On a class of operators, Math. Z. 124 (1972), 199-203

4. I. H. Sheth, On a conjecture of Istrătescu, J. Indian Math. Soc. 38 (1974), 337-338.

Department of Mathematics, Fernuniversität, Postfach 940, 5800 Hagen, Federal Republic OF Germany 